ORIGINAL PAPER

\title{
The RELATIONSHIP BETWEEN PD-L1 EXPRESSION AND TUMOR \\ DRIVER GENE MUTATIONS AND SURVIVAL IN NON SMALL CELL LUNG CARCINOMA
}

\author{
Kin Iong Chan ${ }^{1}$, Hong Ting Vong ${ }^{1}$, Wai Kuou $\mathrm{Chu}^{2}$, Kun $\mathrm{Cheng}^{3}$, Xue Yun Zhong ${ }^{4}$, \\ JiAN Ming Wen ${ }^{1}$
}

\begin{abstract}
${ }^{1}$ Department of Pathology, Kiang Wu Hospital, Macao Special Administrative Region, China
${ }^{2}$ Department of Surgery, Kiang Wu Hospital, Macao Special Administrative Region, China

${ }^{3}$ Department of Internal Medicine, Kiang Wu Hospital, Macao Special Administrative Region, China

${ }^{4}$ Department of Pathology, Medical School of Jinan University, Guangzhou 510632, China
\end{abstract}

\begin{abstract}
To explore the relationship between PD-L1 expression and gene mutations and survival. PD L1, ALK and MET protein expression were detected by immunohistochemistry, and EGFR gene mutation by RT-PCR in 209 cases of NSCLC. The correlations between PD-L1 expression and gene mutations, clinicopathological features and survival was analyzed. PD-L1 was expressed in 99/209 cases (47.4\%) of NSCLC, including score $1(\geq 1 \%$ to $<5 \%) 23$ cases $(11 \%)$, score 2 ( $\geq 5 \%$ to $<50 \%) 36$ cases $(17.2 \%)$, and score $3(>50 \%) 40$ cases $(19.1 \%)$. There were 89 cases $(42.6 \%)$ of EGFR mutation, $12(5.7 \%)$ of ALK and 90 (43.1\%) of MET protein positive. PD-L1 positive expression occurred more frequently in men and non-adenocarcinoma, and was negatively correlated with EGFR mutation and histological differentiation of NSCLC. PD-L1 expression was concordant in primary and metastatic cancers. There was no any effect of PD-L1 expression on overall survival of patients with NSCLC. These results suggested that PD-L1 expression is not an independent risk factor for survival of patients with NSCLC. Because of mutual complementarities of PD-L1 expression and EGFR mutation in NSCLC, both should be simultaneously detected for the patients to achieve eligible treatments.
\end{abstract}

Key words: NSCLC, PD-L1, EGFR; survival.

\section{Introduction}

Lung cancer is worldwide the most morbidity and mortality cancer. According to cancer statistics, there were 234390 new cases and 158080 deaths of lung and bronchus cancer in Unite States, occupying the second and first position of all cancer incidence and mortality respectively in 2016 [1]. In Macau, the highest incidence and mortality of this cancer has lasted for more than a decade [2]. Most lung cancers $(90 \%)$ are non small cell lung carcinoma (NSCLC). Advanced NSCLC, if left untreated, has a poor prognosis. The median survival is about 4 to 5 months, and the 1-year survival rate is less than 10\% [3]. Thus, developing effective treatments are critical for this cancer. Excitingly, many cell signaling pathways initiated by growth factor receptors were found. Multiple genes involved in those pathways underwent mutations, which enabled the tumor cell proliferation, migration and metastasis. These mutated genes in NSCLC are referred as oncogenic driver genes. To date, numerous small molecular inhibitors and monoclonal antibodies have been developed to target those mutation sites and then block the 
signaling pathway of cell growth. Another hot spot for the treatment of NSCLC is immunotherapy, which prevents the cancer cells from immune escape. Both targeted and immune therapies have achieved great success in prolonging overall survival of patients with advanced NSCLC [4]. However, the clinical application of both therapies has to be based on detection of gene mutations and immune checkpoint protein PD-L1 in the tumor cells from the NSCLC patients. Besides, understanding the relationship between gene mutations and PD-L1 expression also help in clinical option for both therapies.

To our knowledge, it still lacks information of PDL1 expression in NSCLC and its correlation with gene mutations in Macau.

\section{Material and methods}

\section{Clinical data}

After approval by the hospital research ethical committee, a total of 209 cases of NSCLC were collected from the archive of Department of Pathology, Kiang Wu Hospital in Macao from January 2007 to December 2017. All patients were informed with the aim of the study and expressed their consent. There were 114 males and 95 females. The age ranged from 33-94 year old (mean age 62 years). According to the 2015 WHO classification, among the cases 196 were adenocarcinomas, 13 non-adenocarcinomas including 6 squamous cell carcinomas, 2 poorly differentiated carcinomas, 3 lymphoepithelioma-like carcinomas, 1 sarcomatoid carcinoma and 1 adenosquamous carcinoma. The tumor tissue or cells were obtained from paraffin sections of pathological specimens or cell blocks of cytological specimens including 123 cases of biopsy or surgical resection, 73 cases of pleural effusion, and 13 cases of fine needle aspiration. Among the 209 cases, 67 were smokers and 142 non-smokers. There were 131 cases with lymph node metastasis and 78 without metastasis. In histological grading, there were 8 well-, 116 moderately-, and 85 poorly-differentiated carcinomas. Tumor stage was defined by the 8th edition of American Joint Committee on Cancer. There were 69 cases of stage I, 21 stage II, 18 stage III and 101 stage IV. All cases were not treated with chemoradio-, immuno- or targeted therapy before sampling and detection.

A total of $186(89 \%)$ patients were followed up. Overall survival time, in months, was measured from the date of pathological diagnosis to the latest follow-up or the date of death. Ninety five were still alive at the end of the follow-up, the remains died from tumor or complications.

All the following experiments were performed with paraffin sections including from tissue blocks of surgical and biopsy specimens and cell blocks of pleural effusion and fine needle aspiration.

\section{DNA extraction}

Tumor cell enriched area in the hematoxylin and eosin stained section was labeled under microscope. It was essential to ensure more than $5 \%$ tumor cells in the background cells to overcome the limit of detection. The corresponding paraffin-embedded tissue or cell block was then serially cut into 3 to 10 slides. The tumor cells in the labeled area was scraped, and digested with proteinase $\mathrm{K}$ overnight. After paraffin dissolution at $99^{\circ} \mathrm{C}$ for 10 minutes, the cells were centrifuged at $10500 \mathrm{~g}$ for 4 minutes at $4^{\circ} \mathrm{C}$, ice bath for 10 minutes to solidify the upper paraffin layer. The cells at bottom were aspirated and the DNA was extracted by QIAamp DNA FFPE Tissue Kit (Qiagen, UK).

\section{EGFR mutation assay}

EGFR mutation was detected by using EGFR RGQ PCR Kit (Qiagen, UK) and ARMS technique. First of all, the reaction mixture was prepared. Each sample was configured with 8 premixes containing different primers and probes. The PCR reaction system was $25 \mu \mathrm{l}$, including $19.5 \mu \mathrm{l}$ of the premix, $0.5 \mu \mathrm{l}$ of the Tap enzyme, and $5 \mu \mathrm{l}$ of the sample DNA (concentration of about $2 \mathrm{mg} / \mathrm{L}$ ). Positive (provided by the kit) and negative (deionized water) controls were set for each PCR reaction. The PCR reaction was pre-denatured at $95^{\circ} \mathrm{C}$ for 15 minutes, then denatured at $95^{\circ} \mathrm{C}$ for 30 seconds, and annealed and extended for 60 seconds at $60^{\circ} \mathrm{C}$ for a total of 40 cycles. Fluorescent signals (including the FAM signal labeled with the mutated gene and the HEX signal amplified by the plasmid) were collected after the end of each cycle extension. The PCR reaction was carried out in a real-time fluorescence quantitative PCR machine and a Rotor Gene Q (Qiagen, UK), and amplification curve in the PCR reaction was analyzed according the guide line of the test kit. The technique could detect 29 types of EGFR mutants in exon 18 to 21.

\section{Immunohistochemistry}

Sections ( $3 \mu \mathrm{m}$ thick) were cut from paraffin-embedded tissue or cell blocks of the tumor or cytology and mounted on microscope slides. Immunohistochemistry was carried out by Benchmark XT automatic staining machine (Ventana, Tucson, AZ) with Ultraview detection system. PD-L1 was detected by using PD-L1 (SP263) rabbit monoclonal antibody (Ventana, Tucson, AZ). Positive and negative controls were set for each test. The staining result was interpreted according to Ventana's interpretation guidelines. Discontinuous, circumferential or basal membrane staining in any intensity of the tumor 

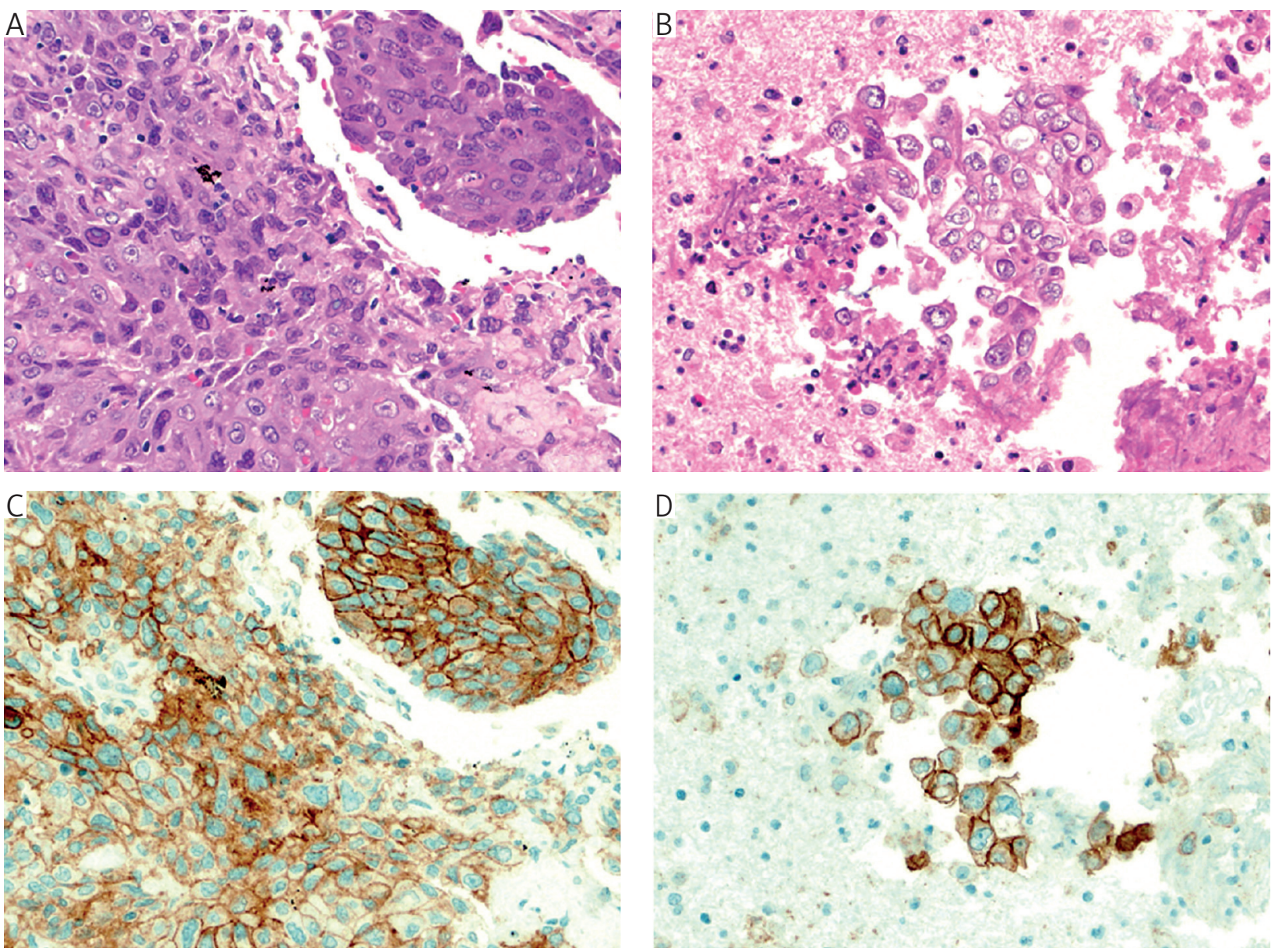

Fig. 1. The staining intensity of PD-L1 in primary and secondary tumor. In this case, the staining intensity of PD-L1 in NSCLC cells is similar to that in the tumor cells of pleural effusion. A) NSCLC tissue, HE staining; B) Tumor cells of pleural effusion, HE staining; C) PD-L1 immunoreaction of NSCLC tissue; D) PD-L1 immunoreaction of tumor cells of pleural effusion (cell block section)

cells were regarded as PD-L1 positive. According to the percentage of positive cells, the immunoreaction was divided into score $0(<1 \%)$, score $1(\geq 1 \%$ to $<5 \%)$, score $2(\geq 5 \%$ to $<50 \%)$, score $3(\geq 50 \%)$. Score 1 to 3 were considered as positive, and score 0 as negative.

For MET protein immunoreaction, c-MET (SP44) rabbit monoclonal antibody (Ventana, Tucson, Arizona) was used. The result was scored according to the immunostaining intensity and ratio of the tumor cells. Complete unstaining was defined as score 0 ; more than $50 \%$ of tumor cells expressed moderate but $<50 \%$ weak positive as score $1 ;<50 \%$ of tumor cells expressed strong but $>50 \%$ moderate positive as $2 ;>50 \%$ of tumor cells expressed strong positive was scored as 3 . Score 0 or $1+$ were regarded as c-MET negative, score 2 and 3 as positive. This scoring method was based on MetMAb criteria [5].

ALK (D5F3) rabbit monoclonal antibody (Ventana, Tucson, AZ, USA) was used to detect ALK protein in the tumor cells with a positive control for each assay. According to the Ventana's interpreta- tion guidelines, complete unstaining was defined as negative. If there was a strong granular cytoplasmic staining in the tumor cells regardless any percentage, the result was regarded as positive.

\section{Statistical analysis}

All statistical analysis was performed using SPSS21.0 software (SPSS, Chicago, Illinois) for Windows. The chi-square was used for rate comparison. The survival curve was determined using Kaplan-Meier method. Multivariate Cox analysis model was applied only when the result of univariate analysis has a statistical significance. The $\mathrm{p}$ values of less than 0.05 were considered statistically significant.

\section{Results}

\section{Gene mutation}

EGFR mutation rate in NSCLC was $42.6 \%$ (89/209). Exon 19 deletion was the most common 
Table I. The relationship between the scores of PD-L1 and clinicopathologic features

\begin{tabular}{|c|c|c|c|c|c|}
\hline \multicolumn{5}{|c|}{ Clinicopathologic PD-L1 intensity (\%) } & \multirow[t]{2}{*}{$\mathrm{P}$} \\
\hline Features & SCORE 0 & SCORE 1 & SCORE 2 & SCORE 3 & \\
\hline \multicolumn{6}{|l|}{ Gender } \\
\hline $\mathrm{M}$ & $51(44.7)$ & $13(11.4)$ & $24(21)$ & $26(22.8)$ & 0.075 \\
\hline $\mathrm{F}$ & $59(62.1)$ & $10(10.5)$ & $12(12.6)$ & $14(14.7)$ & \\
\hline \multicolumn{6}{|l|}{ Age } \\
\hline$\geq 62$ & $60(53.6)$ & $8(7.2)$ & $19(17)$ & $25(22.3)$ & 0.204 \\
\hline$<62$ & $50(51.5)$ & $15(45.5)$ & $17(17.5)$ & $15(15.5)$ & \\
\hline \multicolumn{6}{|l|}{ Smoke } \\
\hline Yes & $80(56.3)$ & $16(11.3)$ & $23(16.2)$ & $23(16.2)$ & 0.326 \\
\hline No & $30(44.8)$ & $7(10.4)$ & $13(19.4)$ & $17(25.4)$ & \\
\hline \multicolumn{6}{|l|}{ Type } \\
\hline Ad & $107(54.6)$ & $21(10.7)$ & $33(16.8)$ & $35(17.9)$ & 0.137 \\
\hline Non Ad & $3(23.1)$ & $2(15.4)$ & $3(23.1)$ & $5(38.5)$ & \\
\hline \multicolumn{6}{|l|}{ Differentiation } \\
\hline Well & $6(75)$ & $0(0)$ & $1(12.5)$ & $1(12.5)$ & $0.023 *$ \\
\hline Moderately & $61(52.6)$ & $16(13.8)$ & $26(22.4)$ & $13(11.2)$ & \\
\hline poorly & $43(50.6)$ & $7(8.2)$ & $9(10.6)$ & $26(30.6)$ & \\
\hline \multicolumn{6}{|l|}{ Stage } \\
\hline I & $39(56.5)$ & $9(13)$ & $13(18.8)$ & $8(11.6)$ & 0.664 \\
\hline II & $12(57.1)$ & $2(9.5)$ & $4(19)$ & $3(14.3)$ & \\
\hline III & $11(61.1)$ & $1(5.6)$ & $3(16.7)$ & $3(16.7)$ & \\
\hline IV & $48(47.5)$ & $11(10.9)$ & $16(15.8)$ & $26(25.7)$ & \\
\hline \multicolumn{6}{|l|}{ LN Metastasis } \\
\hline No & $44(56.4)$ & $9(11.5)$ & $15(19.2)$ & $10(12.8)$ & 0.354 \\
\hline Yes & $66(50.4)$ & $14(10.7)$ & $21(16)$ & $30(22.9)$ & \\
\hline \multicolumn{6}{|l|}{ EGFR mutation } \\
\hline No & $51(42.5)$ & $12(10)$ & $25(20.8)$ & $32(26.7)$ & 0.001 \\
\hline Yes & $59(66.3)$ & $11(12.4)$ & $11(12.4)$ & $8(9)$ & \\
\hline \multicolumn{6}{|l|}{ MET protein } \\
\hline No & $84(70.6)$ & $9(7.6)$ & $14(11.8)$ & $12(10.1)$ & 0.000 \\
\hline Yes & $26(28.9)$ & $14(15.6)$ & $22(24.4)$ & $28(31.1)$ & \\
\hline \multicolumn{6}{|l|}{ ALK protein } \\
\hline Negative & $102(51.8)$ & $21(10.7)$ & $35(17.8)$ & $39(19.8)$ & 0.530 \\
\hline Positive & $8(66.7)$ & $2(16.7)$ & $1(8.3)$ & $1(8.3)$ & \\
\hline
\end{tabular}

$M$ - male; $F$-female; $A d$-adenocarcinoma; $L N$ - lymph node

*The $P$ value is from comparison of score 0 , combination of score 1 and 2, and score 3

mutation, accounting for $44.9 \%$ (40/89). The less common was L858R mutation in exon 21 for $42.7 \%$ (38/89), followed by insertion mutation in exon 20 for $6.7 \%(6 / 89)$, and G719S in exon 18 for $2.2 \%(2 / 89)$, L861Q in exon 21 for $1.1 \%$ (1/89). There were two cases with double mutations; one was G719X/S768I in exon 18 and 20, another T790M/L858R in exon 20 and 21.

\section{Immunohistochemistry}

Of the 209 cases, $99(47.4 \%)$ were positive for PD-L1 protein, including score $1(\geq 1 \%$ to $<5 \%)$ 23 cases $(11 \%)$, score $2(\geq 5 \%$ to $<50 \%) 36$ cases (17.2\%), and score $3(>50 \%) 40$ cases $(19.1 \%)$.

In all cases, merely 12 cases were positive for ALK protein, accounting for $5.7 \%$, while 90 cases were positive for MET protein, accounting for $43.1 \%$. 
Table II. Kaplan-Meier univariate regression analysis in prediction of overall survival

\begin{tabular}{lccc}
\hline \multirow{2}{*}{ VARIATE } & \multicolumn{2}{c}{ Univariate ANALYsis } & P \\
\cline { 2 - 3 } & HazARD Ratio & $95 \%$ CI & 0.171 \\
\hline Gender & 0.751 & $0.499-1.132$ & 0.361 \\
\hline Age & 0.827 & $0.550-1.243$ & 0.383 \\
\hline LN metastasis & 1.224 & $0.777-1.928$ & 0.979 \\
\hline Stage (I-II : III-IV) & 0.994 & $0.638-1.549$ & 0.411 \\
\hline Differentiation & 0.826 & $0.524-1.302$ & 0.287 \\
\hline Well: Moderately & & & 0.598 \\
\hline Well: Poorly & 0.627 & $0.266-1.481$ & 0.148 \\
\hline PD-L1 protein & 0.883 & $0.555-1.403$ & $0.489-1.114$ \\
\hline LN - lymph node & 0.738 & &
\end{tabular}

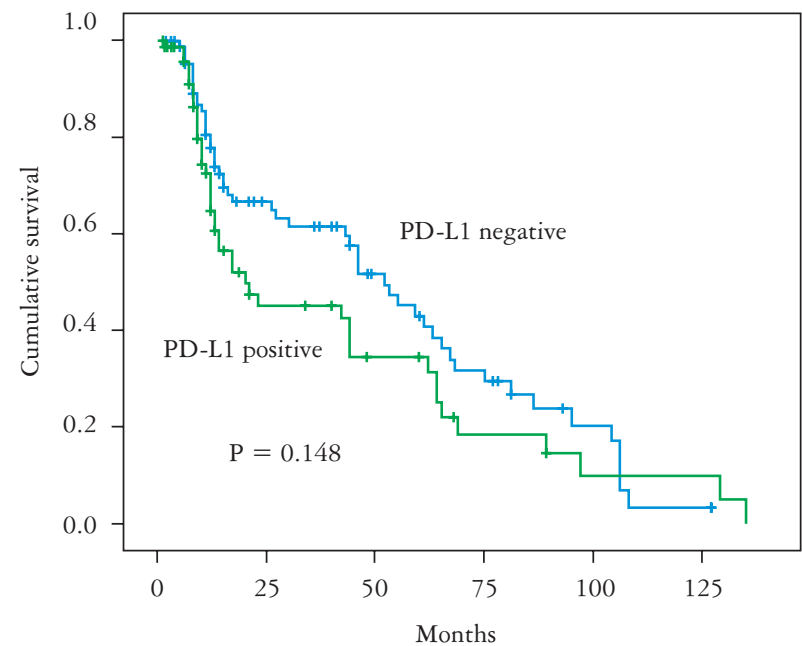

Fig. 2. Cumulative survival in NSCLC patients with PDL1 positive and negative expression

\section{PD-L1 expression in primary and metastatic NSCLC in a same patient}

Ten cases of primary and metastatic cancer specimens were simultaneously obtained in this group of patients studied. Five cases of primary cancer were positive for PD-L1, and their corresponding metastatic tumor cells in pleural effusion were also positive. The rest 5 cases were negative for PD-L1 in primary and metastatic cancer in pleural effusion. The primary and secondary tumor specimens in the same patient are taken in the same time or less than onemonth period before any tumor therapies. In terms of PD-L1 scoring, 3 cases of primary and metastatic cancer were the same in score 3 . In the rest 2 cases, one was score 1 in primary cancer, while score 2 in metastatic cancer, another was score 2 in primary cancer, while score 3 in the metastatic cancer. The PD-L1 protein expression was similar in prima- ry and metastatic cancer in a same patient (Fig. 1), although the score was somewhat different.

\section{Detection of PD-L1 in different specimen types}

Of the 209 specimens examined, 123 were histological specimens (biopsy or resection), and 86 were cytology (pleural effusion or fine needle aspiration). The positive rate of PD-L1 in tissue samples was $43.1 \%$ (53/123), and 53.5\% (46/86) in cytology. There was no statistical difference in the detection rate between both specimen types $\left(\chi^{2}=2.1953\right.$, $\mathrm{p}>0.05)$.

The relationship between PD-L1 expression and clinicopathologic features and gene mutations

The scores of PD-L1 expression were negatively correlated with the tumor differentiation, but not with gender, age, smoking history, clinical stage, or lymph node metastasis. In addition, the scores of PD-L1expression was negatively correlated with EGFR mutation, but positively correlated with MET protein expression. There was no correlation between scores of PD-L1 and ALK protein expression (Table I).

\section{Survival analysis}

In this study, 186 out of 209 cases had adequate follow-up data for the final analysis. The median follow-up time was 12 months (ranged from 1 to 135 months). At the end-point of the study, there were $95(51.1 \%)$ survivors, and the rest 91 (48.9\%) died of tumors or tumor-related complications. There was no any correlation between PD-L1 expression, clinicopathologic features and the patient's overall survival, suggesting PD-L1 expression is not a risk factor for overall survival of patients with NSCLC (Hazard ratio, 0.738, 95\% CI: 0.489-1.114; $\mathrm{p}>0.05$ ) (Table II, Fig. 2). 


\section{Discussion}

In recent years, the application of PD1/PD-L1 monoclonal antibody has achieved unexpected results in immunotherapy of lung cancer. Many studies, including the CheckMate on nivolumab [6], the KEYNOTE on pembrolizumab [7] and the POPLAR and OAK on atezolizumab [8], showed that PD-1/PD-L1 inhibitors had a better effect on NSCLC compared to conventional chemotherapeutic drugs, and could significantly prolong the progression-free and overall survival of the patients. It is no doubt that immunotherapy has become a breakthrough in treatment of NSCLC after targeted therapy. Therefore, understanding the relationship between PD-L1 expression and driving gene mutations is of significance for optional therapies clinically. Tang et al. [9] reported that there was a correlation between EGFR mutation and PD-L1 expression in advanced NSCLC patients treated with EGFR TKIs, but they were irrelevant in early stage of the tumor [10]. Interestingly, PD-L1 expression in tumor and peripheral blood was increased in some patients with mutant NSCLC after TKI drug treatment [11, 12]. Chen et al. [13] deemed that PD-L1 could be regulated by EGFR through the ERK1/2/cJun pathway. Phosphorylation of the mutant EGFR gene initiates the ERK1/2/c-Jun signaling pathway, subsequently, phosphorylation of ERK and c-Jun up-regulated the expression of PD-L1. By using $P I 3 K-A K T$ and MEK-ERK pathway-specific blockers, PD-L1 expression will down-regulated significantly [14]. Similarly, TKI-targeted drugs could also down-regulate the expression of PD-L1 by inhibiting NF- $\mathrm{kB}$ expression in the cells with mutant EGFR [15]. Our results did show that PD-L1 expression was more common in non-mutated EGFR cases of NSCLC. This result was different from Tang et al [9] study with similar research case number and race, but consisted with Yang et al $[10]$ results. In addition, PD-L1 antibodies used in diverse studies were different. Different sensitivity of PD-L1 antibodies could result in controversial results. Therefore, to obtain comparable result accordant detective clone antibody should be used. Nevertheless, the mechanism regulating PD-L1 expression by EGFR mutation in tumor cells remains unclear.

In the KEYNOTE-024 study [7], PD-1 monoclonal antibody (pembrolizumab) and chemotherapy were given to patients with PD-L1 positive rate of $50 \%$. After comparison, immunotherapy had more significant curative effect than chemotherapy (progression-free survival 10.3 vs. 6.0 months and overall survival 30 vs 14.2 months), and the benefit of pembrolizumab treatment even expanded to patients with PD-L1 positive rate of $1 \%$. In our study there were $47.4 \%$ of patients with PD-L1 positive rate of $\geq 1 \%$, thus, almost a half of patients were expected to benefit from anti-PD-L1 therapy. In general, $48.9 \%$ of patients with gene mutations (EGFR or $A L K$ mutation) are suitable for targeted therapy, $47.4 \%$ of patients with PD-L1 positive suitable for immunotherapy in all patients with NSCLC studied. Because most NSCLC carry either targeted gene mutation or PD-L1 positive expression, even both in our study, we suggest that both detections should be performed in order to obtain the most eligible treatment for the patients with NSCLC.

In addition, we found that the clinicopathological features in PD-L1 positive patients was significantly different from the patients with mutant EGFR in NSCLC. Mutation of EGFR genes occurred more common in female, adenocarcinoma, non-smokers and well/moderately histological differentiated tumor as showed in our previous study [16], while PD-L1 expression was more common in male, non-adenocarcinoma, and poorly histological differentiated tumor. Cha et al. [17] deemed that such clinicopathological features could be used as pre-test predictors. Furthermore, we found that PD-L1 expression was positively correlated with MET, but not with ALK protein expression. Therefore, the status of MET and ALK protein expression can also be used as predictors for PD-L1 expression.

The patients with NSCLC may be concerned about what kinds of specimens are suitable for detection of driver gene mutation and PD-L1 expression. We found in the study a close concordance of PD-L1 expression in diverse specimens from biopsy, surgical resection, pleural cytology and fine needle aspiration, indicating different specimens did not affect the detection result of PD-L1 protein if they contained enough number of tumor cells (at least more than $5 \%$ tumor cells in the background cells). Moreover, we obtained consistent results of PD-L1 expression from primary and metastatic cancer in the same patient, suggesting PD-L1 expression is not affected by stages and sites of the tumor [18]. PD-L1 detection in the tumor cells in pleural effusion will benefit the advanced patients with NSCLC to achieve an immunotherapy.

Finally, our results did not demonstrate any effect of PD-L1 expression on overall survival of patients with NSCLC.

This work was supported by grant of Science and Technology Development Fund (003/2013/A1), Macao Special Administrative Region, China.

The authors declare no conflict of interest. 


\section{References}

1. Siegel RL, Miller KD, Jemal A. Cancer statistics, 2016. CA Cancer J Clin 2016; 66: 7-30.

2. Taskforce for annual report of Macao cancer registry. Which cancers were the most common in 2017? In: Annual report of Macao Cancer Registry, 2017. $1^{\text {st }}$ Edition, Macao, Jun 2019. Available at: http://www.ssm.gov.mo/portal/ Accessed Jan 13 2020.

3. Chen W, Zheng R, Baade PD, et al. Cancer statistics in China, 2015. CA Cancer J Clin 2016; 66: 115-132.

4. Politi K, Herbst RS. Lung cancer in the era of precision medicine. Clin Cancer Res 2015; 21: 2213-2220.

5. Spigel DR, Edelman MJ, Mok T, et al. MetLung Phase III Study Group. Treatment rationale study design for the MetLung trail: a randomized, double-blind phase III study of onartuzumab (MetMAb) in combination with erlotinib versus erlotinib alone in patients who have received standard chemotherapy for stage IIIb or IV Met positive non-small-celllung cancer. Clin Lung Cancer 2012; 13: 500-504.

6. Brahmer J, Reckamp KL, Baas P, et al. Nivolumab versus docetaxel in advanced squamous-cell-non-small cell lung cancer. N Engl J Med 2015; 373: 123-135.

7. Reck M, Rodriguez-Abreu D, Robinson AG, et al. KEYNOTE-024 Investigators. Pembrolizumab versus chemotherapy for PD-L1-positive non-small-cell lung cancer. N Engl J Med 2016; 375: 1823-1833.

8. Herbst RS, Baas P, Kim DW, et al. Pembrolizumab versus docetaxel for previously treated, PD-L1-positive, advanced non-small-cell lung cancer (KEYNOTE-010): a randomized controlled trial. Lancet 2016; 387: 1540-1550.

9. Tang Y, Fang W, Zhang Y, et al. The association between PD-L1 and EGFR status and the prognostic value of PD-L1 in advanced non-small cell lung cancer patients treated with EGFR TKIs. Oncotarget 2015; 6: 14209-14219.

10. Yang CY, Lin MW, Chang YL, et al. Programmed cell death-ligand 1 expression in surgically resected stage I pulmonary adenocarcinoma and its correlation with driver mutations and clinical outcomes. Eur J Cancer 2014; 50: 1361-1369.

11. Han JJ, Kim DW, Koh J, et al. Change in PD-L1 expression after acquiring resistance to gefitinib in EGFR-mutant nonsmall-cell lung cancer. Clin Lung Cancer 2016; 17: 263-270.

12. Meniawy TM, Lake RA, McDonnell AM, et al. PD-L1 on peripheral blood $\mathrm{T}$ lymphocytes is prognostic in patients with non small cell lung cancer (NSCLC) treated with EGFR inhibitors. Lung Cancer 2016; 93: 9-16.

13. Chen N, Fang W, Zhan J, et al. Upregulation of PD-L1 by EGFR activation mediates the immune escape in EGFR-driven NSCLC: implication for optional immune targeted therapy for NSCLC patients with EGFR mutation. J Thorac Oncol 2015; 10: 910-923.

14. Ota K, Azuma K, Kawahara A, et al. Induction of PD-L1 expression by the EML4-ALK oncoprotein and downstream signaling pathways in non-small cell lung cancer. Clin Cancer Res 2015; 21: 4014-4021.

15. Lin K, Cheng J, Yang T, et al. EGFR-TKI down-regulates PDL1 in EGFR mutant NSCLC through inhibiting NF- $\kappa$ B. Biochem Biophys Res Commun 2015; 463: 95-101.

16. Chan KI, Vong HT, Sin LF, et al. Relationship between driver gene mutations, their relative protein expressions and survival in non-small cell lung carcinoma in Macao. Clin Respir J 2017; 10: 1-8.

17. Cha YJ, Kim HR, Lee CY, et al. Clinicopathological and prognostic significance of programmed cell death ligand-1 expression in lung adenocarcinoma and its relationship with p53 status. Lung Cancer 2016; 97: 73-80

18. Kim S, Koh J, Kwon D, et al. Comparative analysis of PD-L1 expression between primary and metastatic pulmonary adenocarcinomas. Eur J Cancer 2017; 75: 141-149.

\section{Address for correspondence}

\section{Jian Ming Wen}

Department of Pathology

Kiang Wu Hospital

Macao Special Administrative Region, China

tel. $+853-82958545$

fax: $+853-28347752$

e-mail: wenjm@mail.sysu.edu.cn 